International Journal of HRD Practice, Policy and Research 2017, Vol 2 No 1: 51-66

doi: 10.22324/ijhrdppr.2.105

\title{
Learning to Think and Act with Systems: Improving OD with Soft Systems Methodology
}

\author{
Gillian Felton, National Institute for Health Research, UK \\ Lisa Banton, a City Council, UK \\ Angela Earnshaw, an NHS Foundation Trust, UK
}

\begin{abstract}
Given a context of tough change, Organisational Development practitioners need to be able to innovate as well as develop and implement strategy quickly and efficiently. The key to this is agility - a set of capabilities that can help organisations to rapidly adapt to changing circumstances.

This paper reports on an initiative undertaken by three OD practitioners in three different UK organisations to respond to such pressures and challenge. Critically, the initiative involved them exploring and utilising soft systems methodology (SSM) as an intervention. The paper reports on their initial exposure to SSM and their progress so far. First, the paper presents a brief contextual background regarding OD. This is followed by an explanation of the approach adopted as the learning and application of SSM began. The next stage was to draw rich pictures in relation to the impact upon each of the three OD practitioners, in the context of their chosen organisational problems. The paper concludes with their assessment of the value of this particular pathway of professional development together with some consideration of implications for OD practice more generally.
\end{abstract}

Key Words: organization development, soft systems, managing change, professional development

\section{Introduction}

The field and discipline of Organization Development (OD), for more than 50 years has been concerned with planned and systematic change based on rigorous diagnosis and understanding of problems in order to develop a more effective organization (Cheung-Judge \& Holbeche, 2015). However, it is argued that there is a need to 'reinvigorate OD' at a time when the requirements for change are unprecedented, problems are more difficult and OD practitioners need to show how they can add value (Cummings \& Worley, 2009). Given this context of tough change, OD practitioners need to be able to innovate as well as develop and implement strategy quickly and efficiently. The key to this is agility — a set of capabilities that can help organizations to rapidly adapt to changing circumstances.

Research by the CIPD (2014) suggests that investment in change capability and capacity is essential for organisations that wish to have an ability to effect transformational change. OD, Learning and Development (L\&D) and Human Resource (HR) professionals are critical to this. The CIPD findings advocate that organizations should be able to rely on their OD, L\&D and HR professionals to foresee the need for, and provide expert advice on, change, just as they would expect the finance function in their organization to be able to provide and execute expert advice on complex matters of corporate finance and financial strategy. 
This paper reports on an initiative undertaken by three OD practitioners in three different UK organisations to respond to such pressures and challenge. It seeks to reflect on a professional development pathway with the aim of enhancing their ability to operate in their ever changing environments. Critically, the initiative involved them exploring and utilising soft systems methodology (SSM) as an intervention. The paper reports on their initial exposure to SSM and their progress to date. It reflects on its fitness for purpose in relation to their personal and professional development in the context of OD practice. First, the paper presents a brief contextual background regarding OD. This is followed by an explanation of the approach adopted as the learning and application of SSM began. The next stage was to draw rich pictures in relation to the impact upon each of the three OD practitioners, in the context of their chosen organizational problems. The paper concludes with their assessment of the value of this particular pathway of professional development together with some consideration of implications for OD practice more generally.

\section{OD and SSM}

In a recent review of OD, Burnes and Cooke (2012) have argued that while there has been a large expansion in terms of its theory, practice and reach, OD is not "fundamentally different from the past" (p.1414). Instead there has been a continuous evolution of theory and practice but with a degree of to-ing, fro-ing and even competition between academics and practitioners, exemplifying the dilemma of relevance and/or rigour. The period of the 1940s and 1950s were characterized by relevance and rigour, under the influence of the father figure of action research, Kurt Lewin, whose unfreeze-change-refreeze model of change continues to endure (Bartunek \& Woodman, 2015). This was followed by a period of competition for influence between academe and practitioners but resulting in a degree of supremacy for practitioners during the 1970s and 1980s, particularly the rise of relevant tools and techniques, perfected by consultants and others who could focus on and commercialize their processes of delivery. OD practitioners could gain influence if they could align their work with bigger projects such as Total Quality Management, Business Process Reengineering and Transformational Change and Leadership (McLean, 2007). The work of Schein (1988) and others also gained influence with its concern for the diagnosis of problems in organizations which were "fundamentally problems involving human interactions and processes" (p.12) which would require a consultant establishing a relationship with their clients within organizations (Schein, 1999). During the 1990s and into the 2000s, contributions from academe, especially those that presenting a more pluralist, multi-voiced, languaged and storied version of organization life (See Cummings and Worley, 2009) and change management (Ford \& Ford, 2010) sought to emphasize a more rigorous approach to OD, based on theoretical support. Were such voices heard or considered as some organizations pursued a unipolar path toward profit at any means during the late 2000s leading to the Global Financial Crisis? This suggests that OD needs to add critique and reflexivity to its formative values of humanism and democracy if it is to influence future efforts for progress in organizations. As Burnes and Jackson (2011) have found, if the values of those providing change interventions are not aligned with others who need to subscribe to the change, the result can be failure and resistance.

Burnes and Cooke (2012) suggest that there is now an opportunity for synergy to emerge between academe and practitioners that can provide a "process of renewal" (p.1415) for OD. Such synergy can arise from a reconciliation between the competing forces between relevance and rigour as presented by Stokes (2011), shown as Figure 1. 


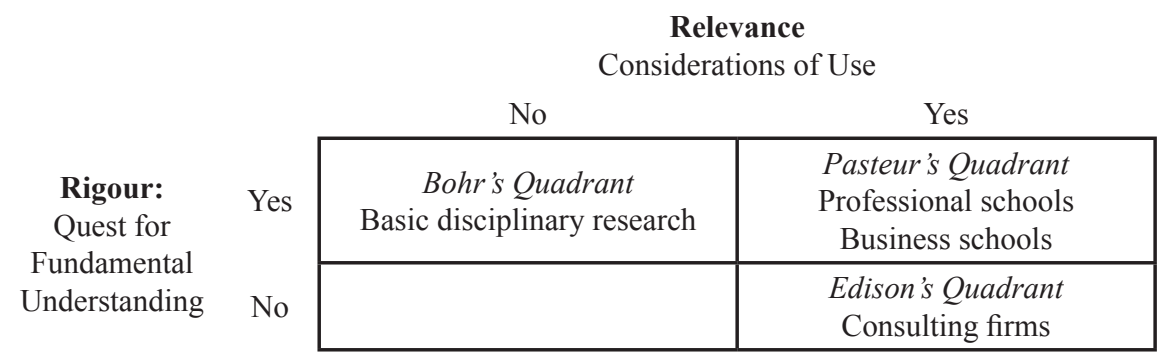

Figure 1: The Choices Between Rigour and Relevance, adapted from Stokes (1997).

Stokes presents three approaches to research and plots them according to their practical use (relevance) on the $\mathrm{X}$ axis and their quest for fundamental understanding (rigour) on the $\mathrm{Y}$ axis.

Bohr's Quadrant, named after the famous atomic physicist Niels Bohr, represents basic research driven by curiosity and not necessarily directed towards solving a practical problem. Whereas Edison's Quadrant named after the legendary inventor Thomas Alva Edison, focuses on practical use and not on the quest for fundamental understanding. He was known for his strong dedication towards solving practical problems rather than his interest in explaining or understanding the phenomenon of a scientific field. Finally, Pasteur's Quadrant where the fundamental understanding gained out of research is used to develop a practical application useful to the society.

It is interesting that Pasteur's quadrant includes business schools, perhaps a reflection of an intent which became lost in the production of theory as an end in itself, or worse, theories that were 'amoral' and could promote 'bad' practices (Ghoshal, 2005). However, it is quite possible for business schools and the academics within them to find a way to work with practitioners in general and OD practitioners in particular which reconciles rigour with relevance. To do this, academics need to escape from the trap that disconnects them from key problems, what Maxwell (2011) refers to as knowledge-inquiry. OD practitioners need to in turn raise their heads to see the significant and critical problems that their organizations are facing. To respond, both can shift to what Maxwell calls wisdom-inquiry, concerned with real problems of living relating to what is of value in life, or what Schon (1991) refers to as research practitioners.

\section{Approach Adopted}

Raelin (2015) considers action modes of research as approaches to enquiry to bring about change within communities such as organizations, but which can also generate and divulge knowledge which can inform both practitioners and academics for relevant actions both in their own worlds and/or jointly. In order to pursue a pathway of CPD for their own OD practice, the OD practitioners in question, selected Soft Systems Methodology (SSM) as the mode of research. Their rationale is developed below.

SSM was principally developed within the Department of Systems at Lancaster University during the 1970s in order to challenge the orthodoxy systems understanding, that systems were a first order reality and move to a view of systems as a way of talking and thinking using the idea of system as a metaphor. The difference between these two views was eventually summarized as the difference between 'hard' and 'soft' systems thinking (Checkland, 1985). Because the 
word system is most usually used to refer to part of the real world ('hard'), Checkland and his colleagues suggested further differentiation by the use of the word 'holon' to refer to our systemic thoughts, i.e. we can think with holons about the world which we view as complex, confusing and messy.

Checkland's (1981) original version for the application of SSM was composed of a seven-stage model, and this is shown as Figure 2.

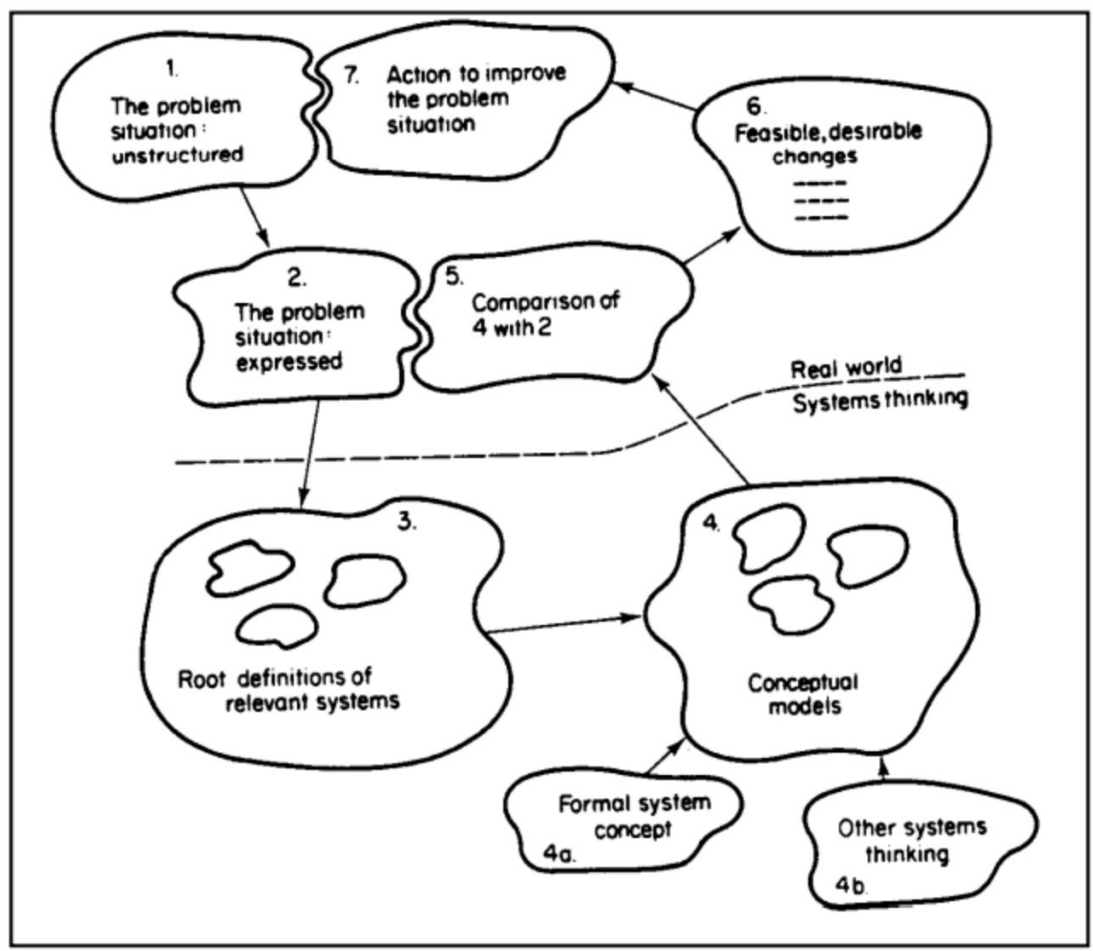

Figure 2: Checkland's Seven-Stage SSM Model

Source: Checkland (1981, p.163)

The value of this framework is that it allows a deepening awareness of a problem by gathering data, reflecting on what is happening before entering an abstract phase of systemic thinking that occurs 'below the line'. This refers to the phases of SSM where abstract thinking, using definitions and models constructed for thinking and talking about the situation, but not as descriptions of the real world. Instead, they could be compared with the real world to stimulate possible changes and actions.

For the exercise reported upon in this paper the approach was to begin with a complex, confusing and messy problem or issue identified by each practitioner and to spend time considering the issue making use of SSM as a model. Through the process of reflecting upon and sharing findings the practitioners were able to form a small action learning set and new possible methods were introduced as appropriate. Each of them was familiar with action learning as a way of helping, supporting and challenging each other by use of questions and with the help of a facilitator. Their reports are presented below. No formal structure is used for these reports as the practitioners 
report what, they individually and in the context of their own problems, found meaningful and of value. The reports present an insight into the activity that took place using modes one and two of the model within a defined time frame of six months.

\section{Reports from Practitioners}

\section{Lisa Banton}

My name is Lisa Banton, Practice Development and Partnership Training Lead for Children's Services at a large City Council.

In terms of context I wanted to reflect at the time on the proposed corporate 'People and Culture Plan' on which Children's services were not consulted and which did not take a systems approach in terms of its development. I wanted to reflect on relationships and processes and identify an inclusive approach to talent and diversity management across a whole system. I also considered the scope of the system i.e. Council wide or city wide.

\section{Current challenge}

The issue that is currently challenging me is how to establish an inclusive talent management system. My awareness map enabled me to identify and reflect on, some of the key themes to consider in the development of the talent management system such as communication, competition and so on. This map also gave me the impetus to challenge partners across Adult Health and Social Care to include the Children's Workforce in the 'One Workforce' Strategy.

On completion of my awareness map, Figure 3, I started a basic reflective log to capture my thought processes and actions arising from the use of the soft systems tools. Subsequently, I collated these themes into an abstract for a discussion paper to present to senior colleagues as an opportunity to share my vision. I wanted to offer an evidence based alternative to the recently produced 'People and Culture Plan' which does not take a Systems thinking or inclusive approach to talent management.

\section{Soft systems tools}

Writing an abstract for a forthcoming HRD Conference inspired me to write a full discussion paper on inclusive talent management. I reviewed the content with my Head of Service following a difficult talent management meeting with the Heads of Strategy and Resources and Human Resources. At the talent management meeting I challenged colleagues about the lack of consultation on the People and Culture Plan and the assumption that this should be 'owned' by Human Resources. I was frustrated by the lack of opportunity to make a contribution. Using soft systems enabled me to identify myself as an actor on the system and to make evidence based recommendations through my discussion paper. A final draft of the paper has been submitted to my chief officer and I am hopeful that some of my ideas will be taken forward to influence the changing shape of Children's Services and perhaps in a wider context.

My awareness map, Figure 3, supported me to explore my initial ideas and knowledge and challenged some of my assumptions. I then took some of the key themes and developed them 
into a rich picture which provides more detail about the relationships between the various directorates or 'communities' within the council as well as external partners. Rich pictures are a key reflective resource for me because they enable me to place myself at the centre which in practice is an uncomfortable place for me to be. This approach challenges me to articulate my vision for change and increases my capacity to influence through sharing my ideas.

\section{Awareness Map \\ Lack of Support, Structure, consistency \\ Talent Management System}

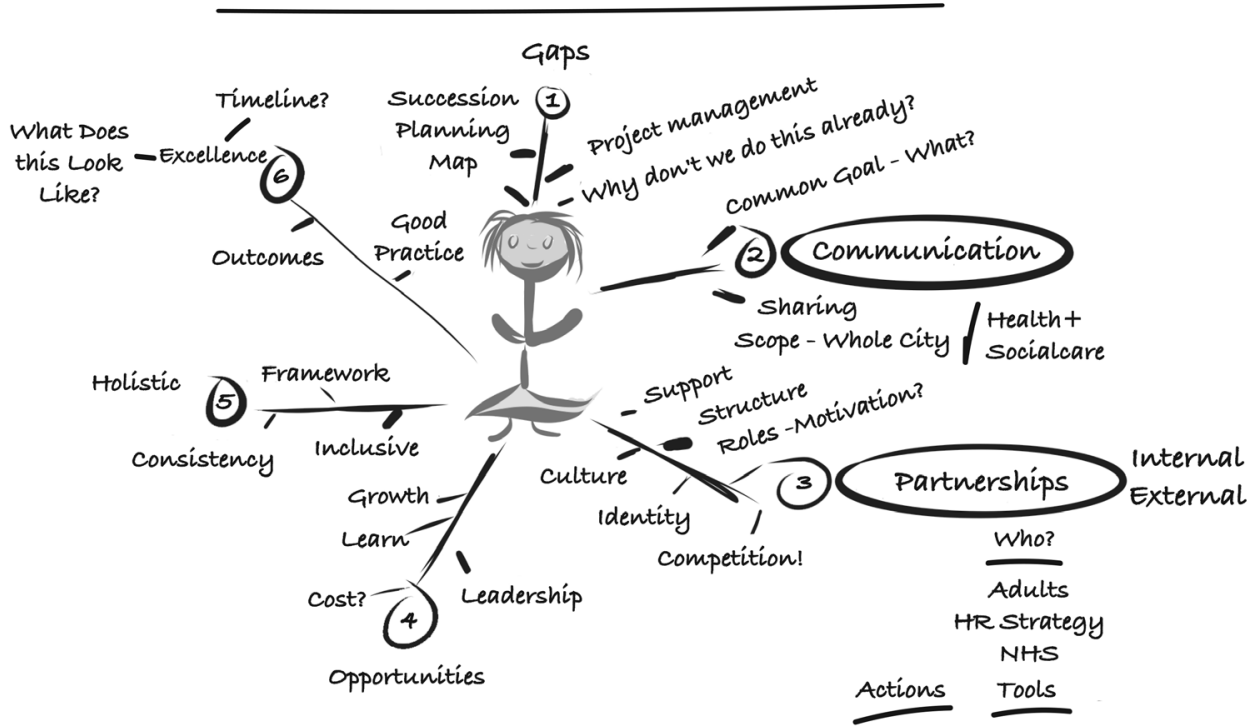

Figure 3: Awareness Map

Before completing my rich picture, Figure 4, I consulted with the Head of Service for Information Management and Technology to gain an additional perspective on political and economic influences on relationships between the directorates. This was a really useful conversation as my colleague challenged some of my assumptions, what I thought I knew, about the corporate view of my own directorate within the council.

Children's Services has direct contact with external customers whereas the Strategy and Resources team only supports internal customers. I came to the conclusion that the directorates hold different roles/provide different functions and therefore different but equally valued forms of support to the chief executive. A large organization such as the Council, values policies and processes as well as creativity and innovation. The question is, can a systems approach successfully incorporate both of these and engage all the stakeholders to achieve positive change? 
As a workforce development lead I am always interested in new ways of learning and tools to support that learning. I am not a visual learner and definitely not an artist but I find the pictorial approach helpful. I am a reflective practitioner and feel that soft systems supported me in that reflection. More importantly I find soft systems particularly effective in enabling me to articulate my ideas, challenge the status quo and confidently offer alternative positions. The use of rich pictures, Figure 4 and awareness maps in particular have provided me with the opportunity to suggest specific actions, such as taking a systems approach to talent management, and therefore influence strategy at a leadership level.

\section{RICHPICTURE SYSTEMS THINKING}

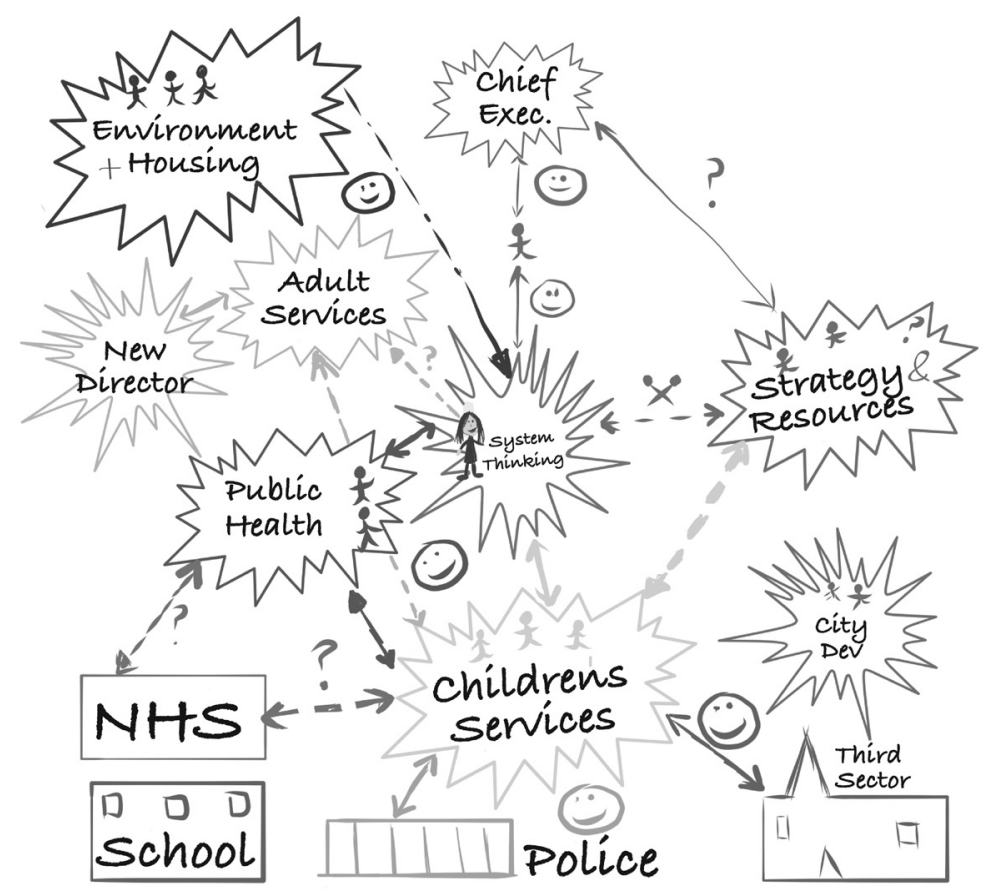

Figure 4: Rich Picture

I enjoy using soft systems as a problem solving tool. It creates the space for viewing change from perspectives within and without the organization. The process encourages me to read and research further into related issues such as leadership which supported the articulation of my vision for inclusive talent management in the council. In particular a book entitled The Search for Leadership by William Tate was very valuable.

I find the action learning sessions a very interesting and supportive part of my reflective learning. I feel that I respond well to exposure to bite sized theoretical information alongside practical workplace challenges. Being able to share frustrations, ideas and interests and develop individual solutions collectively is something I have benefited from significantly. I find systems thinking and soft systems tools very 'human' as well as practical. This means that they are an effective 
tool in delivering positive change. I am constantly frustrated by the de-humanising impact of unnecessary bureaucratic processes and systems. At a time of significant change within my organization I will be seeking out other systems thinkers and leaders in order that we achieve democratic, positive and sustainable change.

\section{Gillian Felton}

I am Gillian Felton, Head of People Development, Engagement and Wellbeing at the National Institute for Health Research Clinical Research Network (NIHR CRN).

\section{Context of the current issue}

The National Institute for Health Research Clinical Research Network (NIHR CRN) is a managed network for delivering clinical research activity in the NHS. It is an organization with a clearly stated vision "clinical research as an integral part of healthcare for all". It is an organization fresh out of a wholesale re-organization with a challenging agenda ahead.

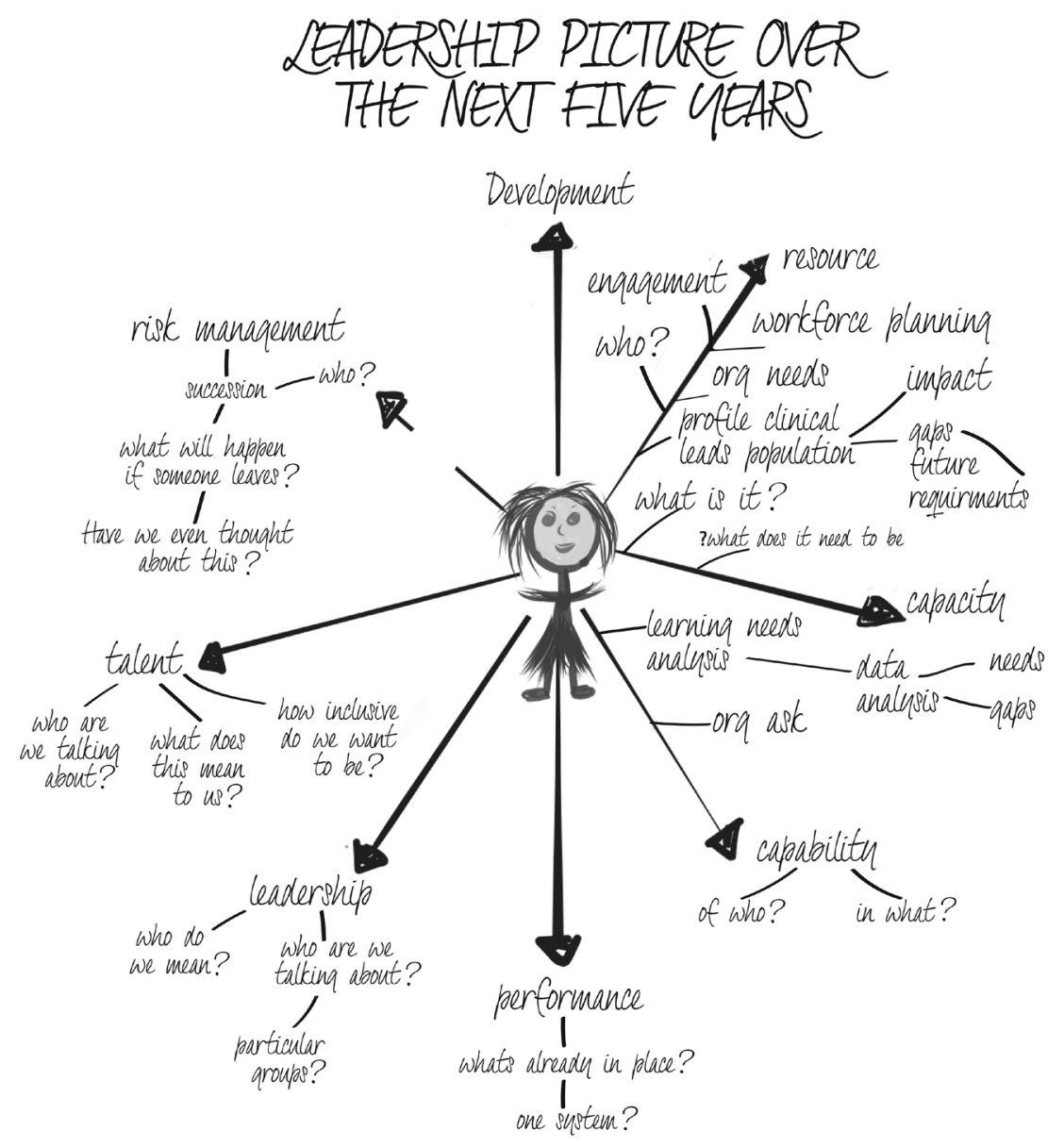

Figure 5: Awareness Map 
The reorganization restructured 102 networks to 15 local clinical research networks and nine coordinating centres streamlined into one. This happened over a two-year timeframe. The restructuring and reshaping have provided many opportunities, not least the chance to review the organization's approach to leadership development and talent management.

\section{Application of soft systems methodology}

When developing awareness for soft systems methodology our attention has to be drawn in the first instance to some 'messy' issue. This allows us to articulate by means of an awareness map all our thoughts, knowledge and general hunches about the issue. My initial awareness map (Figure 5) around leadership development and talent management was made up of headlines and a few questions. It provided the opportunity to visually capture and outline the topics for consideration, who might be involved, what the challenges would be, why we should spend time considering the issue and so on. It allowed for the issue to be seen as a whole in it rawest sense without the need to over describe or answer any of the surfacing questions. Headlines produced headings which in turn generated sub topics and many questions.

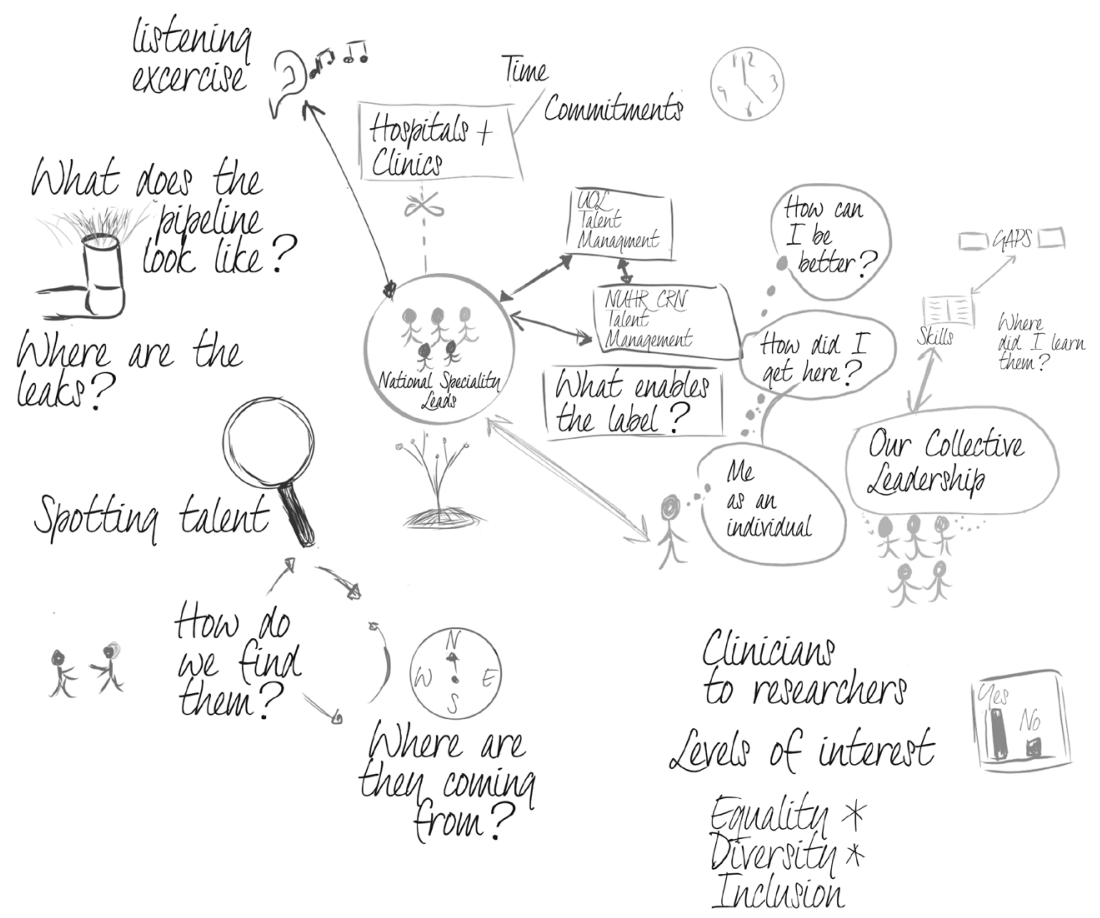

Figure 6: Rich Picture (1)

Whilst the awareness map put the issue on the page it also further confirmed its scale and complexity. This wasn't necessarily a bad thing, quite the opposite in fact as it ensured the magnitude of the task was fully appreciated which in turn led to some considered thinking around how best to tackle it. A one dimensional approach does not work in our networked organization and the prospect of designing an approach to leadership development and talent management for the whole organization had the potential to be overwhelming. 
The awareness map enabled a conversation within the organization which led to refining the approach to attend to a specific client group (National Specialty Leads of which there are 42) rather than attempting to address all client groups at once. The thinking was that by taking a specific group we would be able to test out an approach with the aim of extending it and mapping it onto other groups in the network in the future.

With the client group identified it was then possible to explore the problem in their context. There is a requirement to understand the capacity and capability of the clinical leadership workforce in order to develop a leadership programme that will enable performance now and develop talent for the future. We need a roadmap that will provide the transparency and inclusiveness of opportunity for all.

This was initially done through mind mapping as my preferred method of ideas generation. Soft systems methodology advocates the use of rich pictures as a problem structuring method. Whilst I work in a visual way I do find the concept quite challenging - I would much rather use words than pictures. That said, by generating mind maps in the first instance I was then able to translate them into a rich picture (Figure 6). This suggests that the two approaches complement each other.

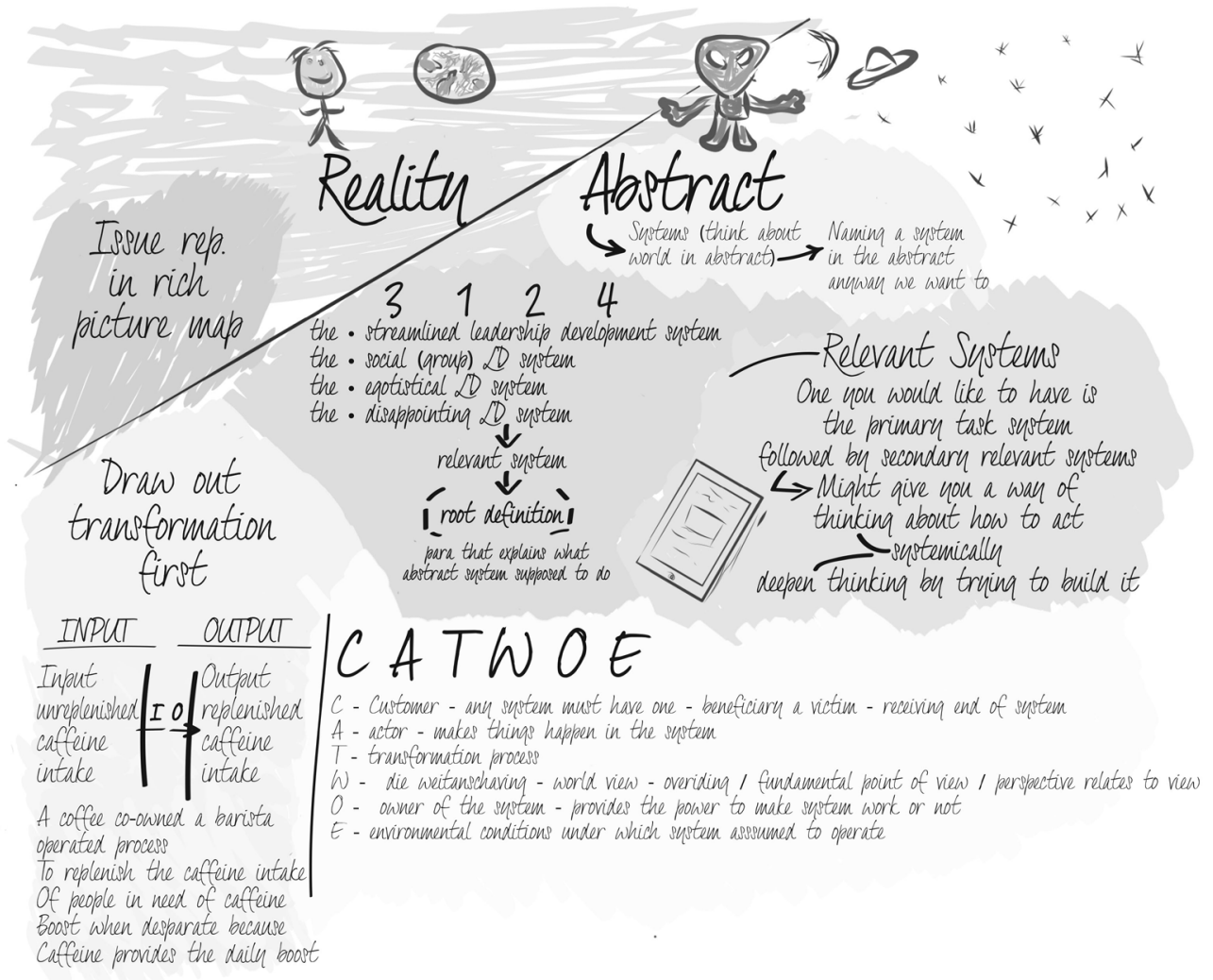

Figure 7: Rich Picture (2) 
With an ultimate aim of a consistent approach across the network to talent management and succession planning underpinned by forward thinking leadership development programmes it was time to intervene in the system of the client group to formulate root definitions. I really needed to understand more about the problem (Figure 7).

In our situation each national specialty lead (the client group) is a stakeholder and therefore has their own perspective of the problem we have posed. I needed to find out what leadership development and talent management meant to them. Using the principles of CATWOE I conducted a listening exercise with pre-determined questions based on four key headings

- You as a National Specialty Lead.

- Leadership and building the collective National Specialty Leadership team.

- Sustaining and nurturing talent.

- Leading clinical research into the future.

This approach enabled me to gather the multiple and contrasting views of the target group and has provided a rich and comprehensive data set. The next step will be to analyse all the data that was captured and move into stage four of soft systems methodology — build conceptual models. Using soft systems methodology is providing a framework to contain the work and give it structure.

\section{My role as OD practitioner using SSM}

As I consider my role as an OD practitioner intervening in the system using SSM I am reminded of the research-practitioner model as described by Schon (1991), and the benefits of connecting research with practice. My reflection of using SSM to tackle this issue highlighted the challenge of making a theory fit with the complex nature of real world reality.

As a methodology it provided a framework to start investigating the problem in question in a different way to the project management approach that our organization would typically adopt. What it did enable was a deep consideration of the 'players' in the system, the human activity and how each of those inform the other and the impact that has on the whole.

The methodology was useful for highlighting the scale of the issue, and its systematic approach allowed for the identification of an initial client group. It provided room for reflection to consider the uncertainty, confusion and messiness of the problem. Being able to articulate how the client group had been selected removed the potential for conflict within the organization. The methodology demonstrated the scale of the issue and the necessity to tackle it a group at a time.

SSM is a way of learning, a way of investigating the breadth and depth of an issue resulting in a plan of action. It is important for us to continue to work through the methodology stage by stage to its conclusion as this will fully test out the validity of its application within the context of the NIHR CRN as a problem solving tool.

\section{Angela Earnshaw}

My name is Angela Earnshaw and I work as the Head of OD within an NHS Foundation Trust. We provide mental health and learning disability care that supports people to achieve the best that they can for their health and wellbeing. 


\section{Using SSM to develop an OD framework}

Facing difficult financial circumstances both in the short and medium terms, my difficult issue concerned the need to develop an OD Framework to take us forwards. Using an awareness map to take a more careful look at the situation, I became aware of how it illustrated the complexity that would be involved. This helped me identify some of the key issues and tensions that needed addressing to take the project forward.

I learned the complexity is part of the work, even though I would prefer simplicity and straight forward connections. I am not comfortable with complexity and ambiguity. I could begin to see a route through and what I could pay attention to. I could take action by holding discussion with respect to some key decisions on resourcing OK. I held consultation discussion with people identified on the map, e.g. leadership development, the Chief Operation Officer and other links to service improvement.

At the time of drawing the map, some issues were unclear and later in the process, as I took action, the map changed or became clearer, either through natural development or my interventions. The map helped me consider issues along a spectrum of what is more certain and what might be a hunch. A key learning point was that it allowed me to realize who I needed to consult with and take on the journey; do I have permission to act - is it in my gift to write this and include in the scope of an OD Framework?

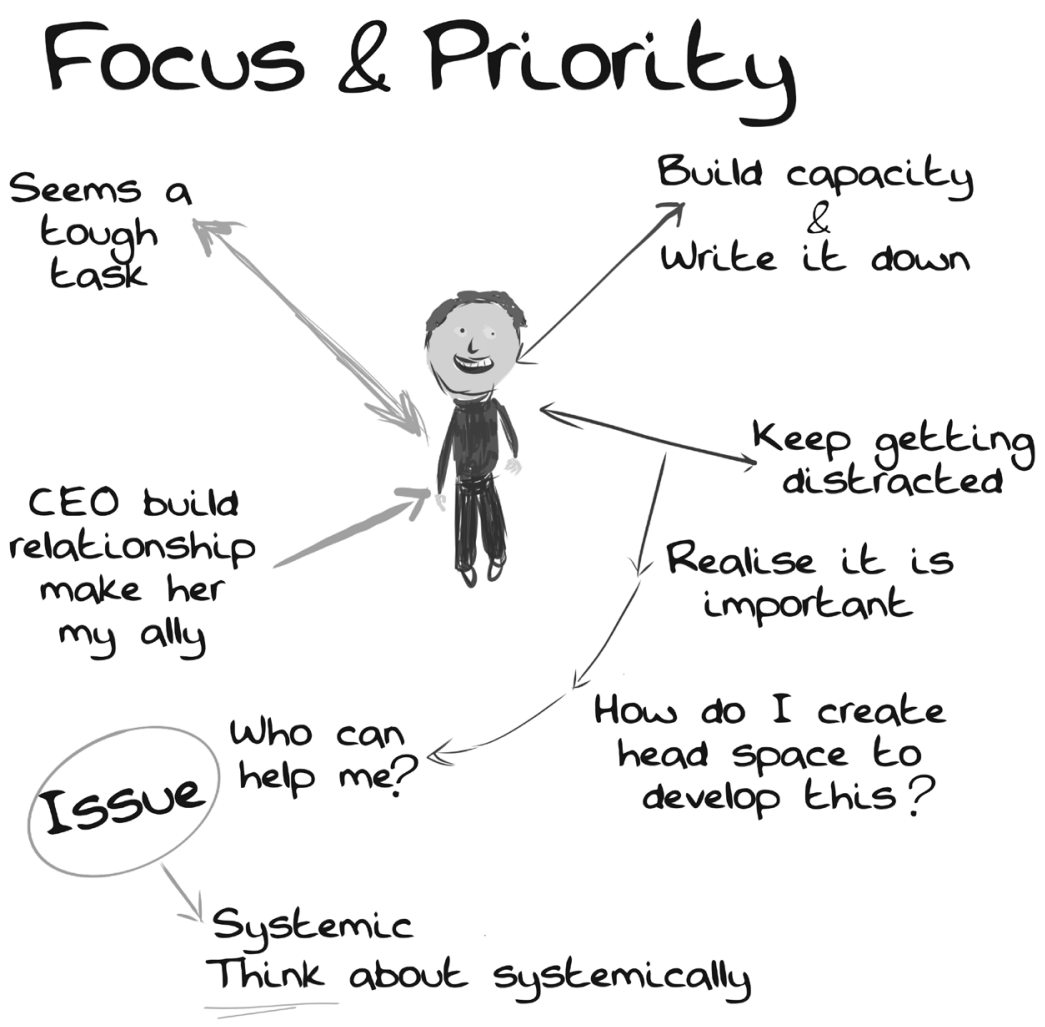

Figure 8: Rich Picture (1) 
I then developed four rich pictures from the map (two of which are illustrated below) to consider:

- The primary focus (Figure 8).

- Ambiguity and general direction (Figure 9).

- Developing competent and trusted leaders.

- Developing OD capacity.

I used the rich pictures to understand key issues and the quality of relationship. I did this in collaboration with key stakeholders to develop ownership and partnership working. This is still a work in progress. The rich pictures help me to identify in detail the important aspect of subsections of the project. In particular, the status of relationships and when work is needed to develop, or repair or change relationships.

\section{Ambiguity-Direction Clearer Now}

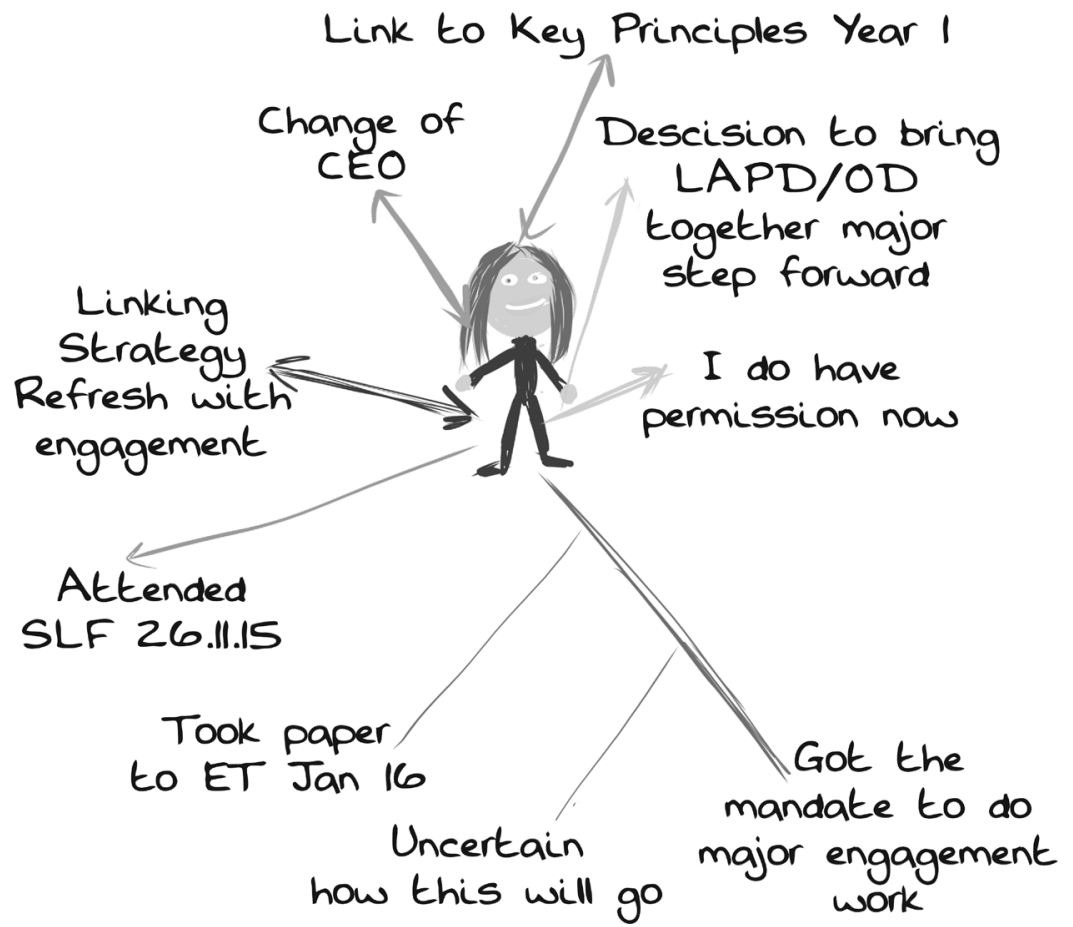

Figure 9: Rich Picture (2)

\section{Conclusions}

According to Schwab (2016), we have begun the fourth industrial revolution during which physical and virtual resources will be combined in new ways to produce a "profound and systemic 
change" (p.9) to our world. In such a world, there will be uncertainty and flux in terms of jobs but whatever their form, there will be a demand for complex problem solving and systems skills. Such a view sits comfortably with the very real organisational problems we as OD practitioners face in our current positions. They are exacerbated by budgetary and resource uncertainty and fundamentally a degree of tension in relation to OD's ability to offer a contribution of value and significance. This said none of the practitioners would have embarked upon this particular pathway of continuous professional development if it was not felt that by learning SSM they would not be able to form plans of action to tackle their respective OD problems.

Although this is very much a project still in process, even by working with awareness and rich pictures, each was able to adopt a way of thinking about complexity that informed taking action, adding depth to their understanding. In this way, they worked along the spectrum of the ideal types of SSM, identified by Checkland (1999) as Mode 1 and Mode 2.

Moving towards Mode 2 SSM, the OD practitioners were able to make use of the tools in particular situations. As managers working on the 'inside', SSM became an ongoing and iterative learning process, the results of which they were able to share with each other as part of action learning. What is apparent is that this feature was important in finding value from what they were able to do and consider SSM favourably, although there were still some key features to consider. Their journey continues.

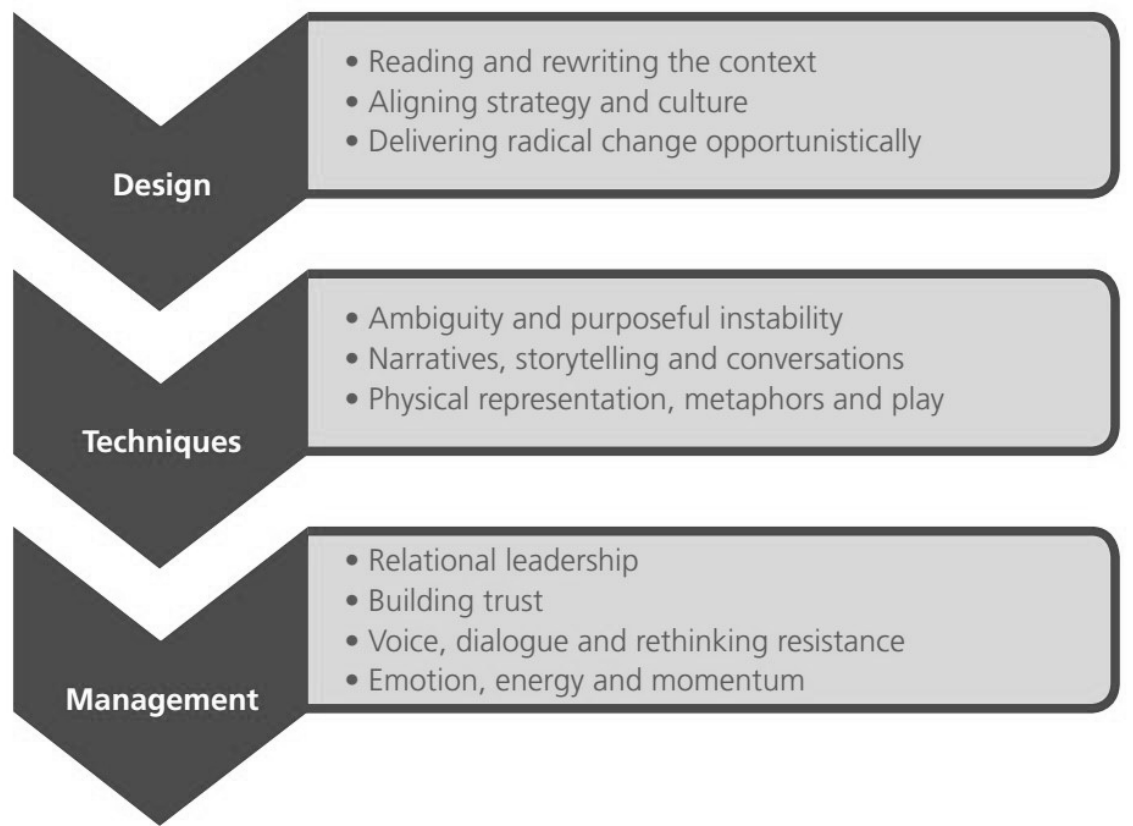

Figure 10: Landing Transformational Change. Source: cipd.org.uk. 2014.

What does their learning and experience of using SSM mean for the OD / HRD / L\&D profession as a whole? Faced with a climate of uncertainty and ambiguity the ability to deploy a range of problem solving tools is critical if any transformation programme is to have a chance of 
being successful. OD, L\&D and HRD practitioners equally have a role in upskilling leaders and managers in organisational change capacity so as to develop those capabilities that will enable organisational flexibility resulting in a more rapid adoption of change.

The CIPD research report (2014) Landing transformational change, sets out ten transformation themes to provide a platform of knowledge on designing, managing and embedding change.

Of particular relevance and linking in with the theme of this paper is the use of physical representation, metaphors and play. They suggest that these three elements are useful at two stages of transformation, firstly as part of the design stage to assist with visioning the new organisation and secondly to make the new culture tangible for the people within the organisation.

To enable the move from the old organisation, a new language, new stories and new behaviours need to be created. Using physical objects and drawings supports these conversations and helps people build a picture of the new organisation. They suggest that using rich pictures encourages involvement as the pictures can be co-drawn allowing the sharing of ideas and a shared understanding of the 'issue'. The visual nature of a rich picture enables a discussion, joint participation and co-ownership. Equally this approach lends itself to a deeper understanding and subsequent ability to recall the messages.

As the demand for transformational change programmes is ever increasing, the demands on the profession to successfully facilitate them also increases. This in turn generates a number of questions practitioners should be asking themselves in terms of their own capability in relation to transformational change and their ability to build change readiness in their organisations.

A one dimensional fixed outlook to transformational change is no longer valid in today's volatile environment, and the successful practitioners will be those who embrace a multi-faceted approach to problem solving and that potentially includes soft systems methodology.

\section{References}

Bartunek, J., \& Woodman, R. (2015). Beyond Lewin: Toward a temporal approximation of organization development and change. Annual Review of Organizational Psychology and Organizational Behavior, 2:157-82.

Burnes, B., \& Cooke, B. (2012) The past, present and future of organization development: taking the long view. Human Relations, 65(11): 1395-1429.

Burnes, B., \& Jackson, P. (2011). Success and failure in organizational change: an exploration of the role of values. Journal of Change Management, 11(2): 133-162.

Checkland P. (1981). Systems Thinking, Systems Practice. Chichester, England: John Wiley \& Sons.

Checkland, P. (1985). From optimizing to learning: a development of systems thinking for the 1990s. Journal of the Operational Research Society, 36(9): 757-767.

Checkland, P. (1999). Systems Thinking, Systems Practice. Wiley, Chichester

Cheung-Judge, M., \& Holbeche, L. (2015). Organization Development: A Practitioner's Guide for OD. Kogan Page.

cipd.org.uk (2014). Landing Transformational change Available at: http://www.cipd.co.uk/binaries/landingtransformational-change_2014.pdf. [Accessed 18 October 2016].

Cummings, T. G., and Worley, C. G. (2009). Organization Development and Change, 8th edn. Mason, OH: South-Western College Publishing. 
Ford, J., \& Ford, L. (2010). Stop blaming resistance to change and start using it. Organizational Dynamics, 39(1): 24-36.

Ghoshal, S. (2005). Bad management theories are destroying good management practices. Academy of Management Learning and Education, 4: 75-91.

Maxwell, N. (2011). We need an academic revolution. Oxford Magazine, 309: 15-18.

McLean, G. N. (2007). Organization Development: Principles, Processes and Performance. San Francisco, CA: Berrett-Koehler.

Raelin, J. (2015). Action modes of research. In L. Anderson, J. Gold, J. Stewart \& R. Thorpe (eds) Handbook of Professional Doctorates in Business and Management, London: Sage

Schein, E. H. (1988). Process Consultation - Volume 1: Its Role in Organization Development, 2nd edn. Reading, MA: Addison Wesley.

Schein, E. H. (1999). Process Consultation Revisited: Building the Helping Relationship. Reading, MA: Addison Wesley.

Schon, Donald A. (1991). The Reflective Practitioner, how professionals think in action. Aldershot: Ashgate Publishing limited.

Schwab, K. (2016). The Fourth Industrial Revolution. Geneva: World Economic Forum.

Stokes, D. E. (2011). Pasteur's Quadrant: Basic Science and Technological Innovation. Brookings Institution Press, 2011

\section{The Authors}

Gillian Felton is Head of People Development, Engagement and Wellbeing at the National Institute for Health Research Clinical Research Network (NIHR CRN), responsible for ensuring the needs of the people are aligned with the needs of the business. The NIHR CRN provides the infrastructure across the whole of England that enables high-quality clinical research to take place in the NHS. She has over 25 years experience working in the HR profession in various roles across a variety of organisations in both the private and public sectors. She is alumni of Roffey Park, the internationally renowned leadership institute based in the UK and Singapore. She is also a Chartered Member of the Chartered Institute of Personnel and Development, the professional body for HR and people development.

Lisa Banton has been the Workforce Development Lead in a large local authority for the past 4 years. She is committed to people development and talent management through a restorative approach to leadership. She led a highly successful culture change training programme in Restorative Practice in 2015 which was part of an award winning programme. She has been part of a multi-agency, organizational development action learning set since April 2013. She graduated in Law in 1994 and has an M.A. in Youth and Community Development.

Angela Earnshaw is Learning and Organizational Development Lead for an NHS Foundation Trust. She is passionate about supporting the development of people, organizations and systems. She has a keen interest in organizational development, coaching and supervision and actively works with colleagues across the city of Leeds to support development that improves health and social care services. She has a master's degree in human resource development. 